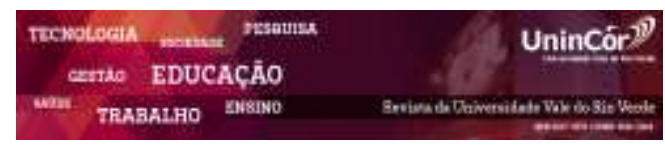

Revista da Universidade Vale do Rio Verde ISSN: 1517-0276 / EISSN: 2236-5362 Vol. 16 | n. 1 | Ano 2018

Isabella Maria de Oliveira Miranda Centro Universitário das Faculdades

Associadas de Ensino isabella.mariamiranda@hotmail.com

Eduarda Zeuri

Centro Universitário das Faculdades

Associadas de Ensino duda.zeuri@hotmail.com

Karolina Tank

Centro Universitário das Faculdades

Associadas de Ensino

kaa.tank@hotmail.com

João Gabriel Barbosa

Centro Universitário das Faculdades

Associadas de Ensino

joaogabrielbarbosa1@gmail.com

Nelson Antônio Filho

Universidade Pontifícia Católica de

Campinas

nelsonpsic@gmail.com

Laura Ferreira de Rezende Universidade de Campinas e Universidade do Estado de São Paulo laura@fae.br

\section{CARACTERIZAÇÃO DA IDEAÇÃO SUICIDA EM ESTUDANTES UNIVERSITÁRIOS}

\section{RESUMO}

Na sociedade moderna, o suicídio tem se tornado cada vez mais recorrente. Portanto, objetivou-se entender e pontuar a prevalência do comportamento de ideação suicida em jovens universitários. Foi utilizado o método sistemático de revisão para a elaboração deste artigo. Com isso, analisou-se a presença de fatores externos associados às causas prevalentes para a ideia de suicídio, estando dentre eles: o abuso de drogas lícitas e ilícitas; pressão da faculdade e conflitos familiares; e, por fim, fatores internos como saúde mental debilitada e depressão. Todos os fatores pré-dispõem o universitário a um comportamento impulsivo. Entretanto, evidenciou-se que a depressão não é um fator exclusivo para a ideia e efetivação do suicídio. Apesar de todos os riscos discutidos, não foi evidenciado uma estratégia de política pública voltada à ideia de suicídio. Dessa maneira, observou-se que os sistemas públicos de saúde não dispõem de fatores que possam prevenir distúrbios associados a ideia de suicídio, assim como também não dispõem de intervenção e promoção da saúde mental à população universitária.

Palavras-chave: Atentado suicida. Adulto jovem. Suicídio universitário. Ideação suicida. Universitários.

\section{CHARACTERIZATION OF SUICIDE IDEA IN UNIVERSITY STUDENTS}

\begin{abstract}
In modern society, the suicide has been turned increasingly recurrent. Therefore, ambitioned to understand and assess the prevalence of suicidal ideation behavior in young university students. The systematic review method was used for the elaboration of this article. Thereby, it was analyzed the presence of external factors associated with the prevailing causes for the idea of suicide, including: the abuse of licit and illicit drugs; College pressure and family conflicts; and, lastly, internal factors such as debilitated mental health and depression. All factors predispose the university student to impulsive behavior. However, it was evidenced that depression is not an exclusive factor to the idea and effectiveness of suicide. Despite all the discussed risks, there was no evidence of a strategy of public policy focused on the idea of suicide. Thus, it was observed that public health systems do not have factors that can prevent disorders associated with the idea of suicide, nor do they have intervention and promotion of mental health to the university population.
\end{abstract}

Keywords: Suicide attack. Young adult. University suicide. Suicidal ideation. University students. 


\section{INTRODUÇÃO}

Sabe-se que o suicídio é uma pratica comum na sociedade. No Brasil, por exemplo, foi constatado que a taxa de suicídio em jovens alcançou 5,4 para cada 100.000 habitantes no ano de 2007, o que representa um índice preocupante. As literaturas apontam que as pressões externas afetam diretamente a vida desses jovens, que apesar de serem bem-sucedidos, como adentrando ao mercado de trabalho, nos cursos técnicos ou no nível superior, apresentam alta prevalência de ideação suicida (DUTRA, 2012).

É senso comum que a depressão esteja intimamente atrelada a tentativa de suicídio, entretanto, autores indicam que existem outros fatores associados, tais quais problemas financeiros, mudança no estilo de vida, pressão familiar, abuso de álcool e/ou droga e direção perigosa intrínsecos ao comportamento suicida. Esses fatores podem estar relacionados ou não à depressão. Porém em muitos casos há a falta de registro sobre o ato suicida, que colaboram para números incertos, pois sabe-se mais sobre as notícias inseridas na mídia informal do que por registros oficiais (DUTRA, 2012). Diante dos dados apresentados tem-se que o objetivo desse estudo é entender e pontuar a prevalência do comportamento de ideação suicida em jovens universitários, por meio de uma revisão sistemática.

\section{MÉTODOS}

Os artigos foram pesquisados nas bases de dados Lilacs, Pubmed, Medline, entre 2006 e 2016 com as seguintes palavras chaves: atentado suicida, adulto jovem, suicídio em universitários, ideação suicida e universitário, cruzados aleatoriamente com palavras em português e inglês.

O primeiro critério de exclusão foi ser resumo, seguindo outros critérios como questões de compreensão da morte, luto, artigos inferiores a 2006, artigos de revisão e revista. O segundo critério de exclusão a partir da leitura completa do artigo, usando os seguintes fatores: jovens de 15 a 35 anos, não universitários, artigos que falam sobre a escala de Beck sem justificativa do porquê da ideação suicida, como observado na Figura 1. 
Figura 1 - Fluxograma de identificação e seleção dos artigos para revisão sistemática sobre a ideação suicida em jovens universitários.

Número de relatos identificados nas bases de dados:

- avaliador 1: 1166

- avaliador 2: 1108

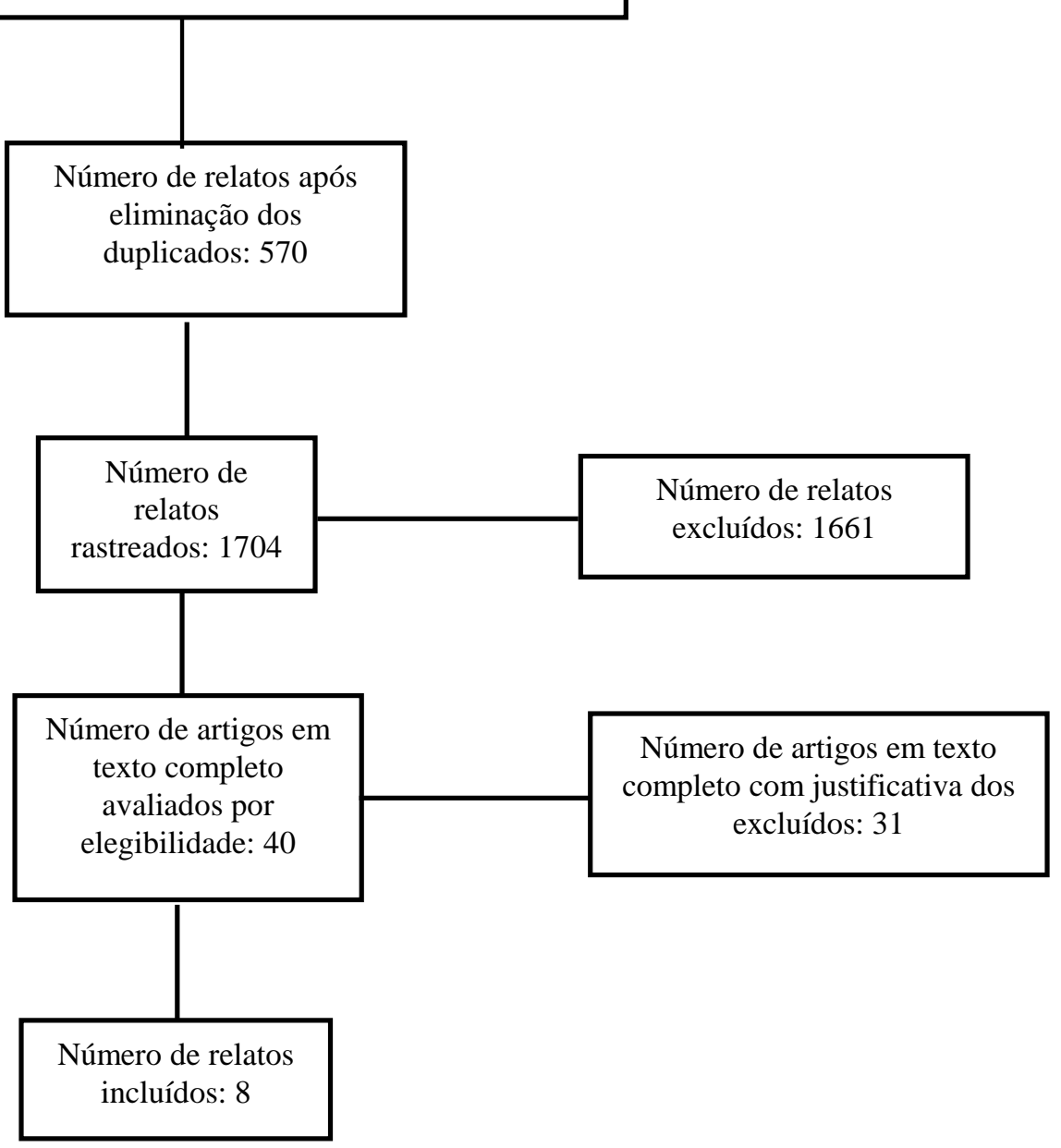

Fonte: tabela elaborada pelo próprio autor com embasamento nos dados fornecidos pelos artigos científicos.

\section{RESULTADOS E DISCUSSÃO}

Foram selecionados 8 artigos com índice de concordância nas bases de dados de 0,95, e os avaliados por elegibilidade 0,81 como observado na Tabela 1 . 
Tabela 1 - Descrição dos artigos selecionados nas bases de dados PubMed e Medline quanto aos autores, métodos, avaliações, resultados e explicações para as inferências.

\begin{tabular}{|c|c|c|c|c|}
\hline Autor (ano) & Método & Avaliação & Resultados & Explicação dos autores \\
\hline Pereira et al; (2015) & $\begin{array}{l}\text { Estudo transversal; } \\
366 \text { estudantes, sendo: } \\
60 \text { estudantes da Faculdade de } \\
\text { Ciências Agrárias e } \\
\text { Veterinárias, } 86 \text { na Escola da } \\
\text { Vida e Ciências Ambientais, } 83 \\
\text { da Faculdade de Ciência e } \\
\text { Tecnologia, e } 137 \text { pela } \\
\text { Faculdade de Ciências } \\
\text { Humanas e Sociais. }\end{array}$ & $\begin{array}{l}\text { Foram realizadas questões } \\
\text { dicotômicas, com "sim" e } \\
\text { "não", e questões de múltipla } \\
\text { escolha a fim de avaliar a } \\
\text { ideação suicida. }\end{array}$ & $\begin{array}{l}12,6 \% \text { dos estudantes } \\
\text { afirmaram ter desejos de } \\
\text { morrer; } \\
5,5 \% \text { afirmam em fazer } \\
\text { algo para tirar suas vidas. }\end{array}$ & $\begin{array}{l}1 \text { em cada } 10 \text { alunos apresentaram ideação } \\
\text { suicida. } \\
\text { A prevalência de ideação suicida é preocupante } \\
\text { em estudantes universitários. } \\
\text { Os serviços de prevenção e de intervenção são } \\
\text { obrigados a intervir neste nível de ensino. }\end{array}$ \\
\hline Baader et al; (2014) & $\begin{array}{l}\text { Estudo transversal; } \\
804 \text { estudantes universitários; } \\
\text { Seleção aleatória de carreiras e } \\
\text { ano acadêmico. }\end{array}$ & $\begin{array}{l}\text { Foram respondidas escalas in- } \\
\text { dividualmente de depressão } \\
\text { (paciente Questionnaire-QSP-9 } \\
\text { Saúde), para o risco de suicídio } \\
\text { (Escala de desesperança de } \\
\text { Beck-HBS) para (Questionário } \\
\text { sobre Transtorno de Humor - } \\
\text { MDQ) transtornos bipolares, } \\
\text { para o álcool e as drogas (DEP - } \\
\text { ado e de auditoria) e para trans- } \\
\text { tornos alimentares (Escala de } \\
\text { diagnóstico para avaliar trans- } \\
\text { torno alimentar EDDS, e res- } \\
\text { tringir Escala-RS). }\end{array}$ & $\begin{array}{l}27 \% \text { dos alunos universitá- } \\
\text { rios foram diagnosticados } \\
\text { com depressão; } \\
10,4 \% \text { transtorno bipolar; } \\
5,3 \% \text { moderado risco } \\
\text { grave de suicídio; } \\
24,2 \% \text { apresentam } \\
\text { problema de álcool; } \\
\begin{array}{l}15,3 \% \text { transtorno } \\
\text { alimentar. }\end{array}\end{array}$ & $\begin{array}{l}\text { A depressão foi significativamente associada com } \\
\text { uma tendência maior a ter um comportamento } \\
\text { alimentar anormal, uso nocivo de álcool e } \\
\text { comportamento autodestrutivo. }\end{array}$ \\
\hline Eskin et al (2016) & $\begin{array}{l}\text { Coorte retrospectivo; } \\
5.572 \text { estudantes universitários; } \\
\text { Quatro zonas Confucionista } \\
\text { (China, Japão); Islâmica (Irã, } \\
\text { Jordânia, Palestina, Arábia } \\
\text { Saudita Arábia, Turquia, Tuní- } \\
\text { sia); língua inglesa (Reino Uni- } \\
\text { do, Estados Unidos) e católica } \\
\text { (Áustria, Itália). }\end{array}$ & $\begin{array}{l}\text { Questionários utilizado auto- } \\
\text { administrado incluia perguntas } \\
\text { sobre comportamento suicida } \\
\text { não fatais, afiliação religiosa e } \\
\text { força da crença religiosa,atitude } \\
\text { de suicídio e individuos } \\
\text { suicidas, orientações de valores } \\
\text { individualitas-coletivo. }\end{array}$ & $\begin{array}{l}28,8 \% \text { dos participantes } \\
\text { tem pensamentos suicidas; } \\
7 \% \text { relatam ter terntado se } \\
\text { matar; } \\
\text { Porcentagens mais altas na } \\
\text { Áustria e Jordânia. } \\
\text { Porcentagens mais baixa } \\
\text { na Arábia Saudita e Itália. }\end{array}$ & $\begin{array}{l}\text { Ideação suicida, tentativa de suicídio e sofrimento } \\
\text { psicológico são comuns em estudantes universitá- } \\
\text { rios. } \\
\text { Suas taxas variam dependendo do contexto } \\
\text { sociocultural. } \\
\text { Atenção dedicadas às necessidades de saúde } \\
\text { mental de jovens adultos. }\end{array}$ \\
\hline
\end{tabular}




\begin{tabular}{|c|c|c|c|c|}
\hline Lamis et al; (2016) & $\begin{array}{l}\text { Coorte retrospectivo; } \\
2.034 \text { estudantes universitários. } \\
\text { A amostra foi composta por } \\
\text { estudantes de primeiro ano }(\mathrm{n}= \\
968) \text {, estudantes de segundo } \\
\text { ano }(\mathrm{n}=491), \text { juniores }(\mathrm{n}= \\
\text { 334) e idosos }(\mathrm{n}=241) .\end{array}$ & $\begin{array}{l}\text { Índice de Problemas do Álcool } \\
\text { de Rutgers (RAPI), RAPI usado } \\
\text { para avaliar as consequências } \\
\text { relacionadas ao álcool, comuns } \\
\text { entre estudantes universitários } \\
\text { (por exemplo, falta de classe, } \\
\text { lutas ou discussões, condução } \\
\text { após beber). }\end{array}$ & $\begin{array}{l}\text { Associação entre sintomas } \\
\text { depressivos e desesperança } \\
\text { foi diminuída entre os alu- } \\
\text { nos com altos } \\
\text { apoíveis de social. } \\
\text { Problemas de álcool foram } \\
\text { associados }\end{array}$ & $\begin{array}{l}\text { O apoio social pode ser uma variável fundamental } \\
\text { para a prevenção do suicídio entre os estudantes } \\
\text { universitários. }\end{array}$ \\
\hline Faria et al; (2014) & $\begin{array}{l}\text { Estudo transversal; } \\
210 \text { universitários }\end{array}$ & 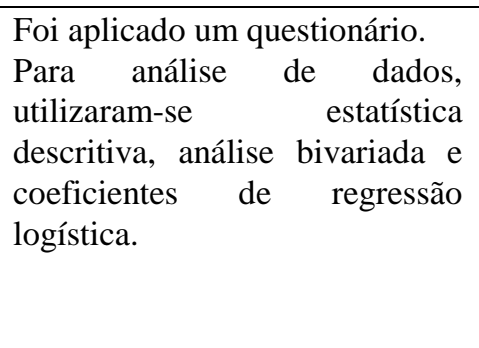 & $\begin{array}{l}40 \% \text { dos estudantes } \\
\text { consumiram álcool; } \\
25 \% \text { apresentaram excesso } \\
\text { de peso; } \\
19 \% \text { utilizaram motocicle- } \\
\text { tas para transporte; } \\
6 \% \text { relataram tentativa de } \\
\text { suicídio. }\end{array}$ & $\begin{array}{l}\text { Comportamentos que colocaram a saúde em risco. } \\
\text { Mais frequentes em homens. } \\
\text { O consumo de álcool foi o comportamento de } \\
\text { risco mais prevalente nessa população. }\end{array}$ \\
\hline Silva et al; (2009) & $\begin{array}{l}\text { Estudo transversal; } \\
989 \text { alunos; } \\
\text { Estudantes de medicina } \\
(\mathrm{n}=603) \text {, enfermagem }(\mathrm{n}=187) \\
\text { e farmácia }(\mathrm{n}=199) \text {. }\end{array}$ & $\begin{array}{l}\text { Escala de Beck para ideação } \\
\text { suicida (BSI), com } 21 \text { itens. } \\
\text { Inventário de Depressão de } \\
\text { Beck (BDI) com } 21 \text { itens. } \\
\text { A medida de depressão foi } \\
\text { associada com a Escala de } \\
\text { Desesperança de Beck (BHS) } \\
\text { com } 20 \text { itens. }\end{array}$ & $\begin{array}{l}\text { Poder estatístico de } 85 \% \text {. } \\
573 \text { dos quais } 78 \% \text { eram } \\
\text { do sexo feminino }(69 \% \\
\text { estudantes de medicina, } \\
94 \% \text { estudantes de enfer- } \\
\text { magem e } 88 \% \text { estudantes } \\
\text { de farmácia). } \\
\text { A Idade média foi de } 22,21 \\
\text { anos. } \\
\text { Não houve diferença } \\
\text { significativa entre os sexos } \\
\text { no BHS e à presença de } \\
\text { ideação suicida. }\end{array}$ & $\begin{array}{l}\text { Presença de desesperança é significativamente } \\
\text { maior nos estudates de medicina em comparação } \\
\text { em enfermagem e a farmácia. }\end{array}$ \\
\hline Shenoy et al; (2015) & $\begin{array}{l}\text { Estudo transversal randomiza- } \\
\text { do; } \\
14.323 \text { alunos. }\end{array}$ & $\begin{array}{l}\text { O instrumento ACHA-NCHA } \\
2013 \text { continha } 66 \text { itens. } \\
\text { Os quais são interessantes: } \\
\text { característica demográfica (por } \\
\text { exemplo, idade, sexo, etnia), }\end{array}$ & $\begin{array}{l}\text { Idade média de } 22,4 \text { ano. } \\
\text { Predominantemente do } \\
\text { sexo feminino. } \\
\text { Agregados: questões } \\
\text { traumáticas, dificuldade }\end{array}$ & $\begin{array}{l}\text { Os alunos de pais solteiros enfrentam demandas } \\
\text { concorrentes e podem enfrentar incidência de } \\
\text { dificuldades de saúde mental, incluindo ideias e } \\
\text { tentativas suicidas. }\end{array}$ \\
\hline
\end{tabular}




\begin{tabular}{|c|c|c|c|c|}
\hline & & $\begin{array}{l}\text { questões traumática ou difíceis } \\
\text { (por exemplo, acadêmicos, } \\
\text { problemas familiares, } \\
\text { financeiro) e saúde mental. }\end{array}$ & $\begin{array}{l}\text { com a morte de um ente } \\
\text { querido, problemas famili- } \\
\text { ares. } \\
\text { Financeiro: alunos de pais } \\
\text { solteiros tem problemas } \\
\text { financeiros. } \\
\text { Saúde mental: estado de } \\
\text { humor negativo ou suicida. }\end{array}$ & \\
\hline Micin et al; (2011) & $\begin{array}{l}\text { Coorte retrospectivo; } \\
460 \text { estudantes universitários. }\end{array}$ & $\begin{array}{l}\text { Transferência de dados a partir } \\
\text { das fichas para o "Protocolo de } \\
\text { Diagnóstico" de cada aluno. } \\
\text { O levantamento de dados durou } \\
\text { três meses, com } 110 \text { horas. }\end{array}$ & $\begin{array}{l}\text { Os resultados mostram } \\
\text { taxas significativas: para } \\
\text { transtornos de adaptação, } \\
\text { humor, ansiedade, } \\
\text { transtornos r de } \\
\text { personalidade, transtornos } \\
\text { alimentares e um histórico } \\
\text { de comportamento suicida } \\
\text { foram encontrados. }\end{array}$ & $\begin{array}{l}\text { Transtornos de ajustamento relacionados a } \\
\text { conflitos familiares ou casal, mudanças de cidade, } \\
\text { conflitos interpessoais estressores psicossociais. }\end{array}$ \\
\hline
\end{tabular}


O presente trabalho visa elucidar os fatores que levam o universitário à ideação suicida. Os seguintes artigos exemplificam as diversas causas para a ideia de morte, buscado entender e assim monitorar condutas de risco à saúde por jovens universitários. Este comportamento é iniciado com o ingresso na universidade, que os torna livres para determinadas escolhas. (FARIA et al, 2014).

O artigo do autor Eskin et al (2016), abrange 12 países, e analisa estudantes universitários que estão inseridos em culturas diversas e condições socioeconômicas diferentes. Incluindo países desenvolvidos e em desenvolvimento, o que leva a refletir sobre as diferentes concepções do significado de suicídio. Nos países árabes existe uma pressão religiosa sobre o suicídio, sendo essa uma prática condenada pela religião. Dessa forma, há uma ocultação dos dados verídicos de suicídio nessa cultura, uma vez que esse ato é considerado uma prática pecaminosa.

De acordo com o autor Pereira et al (2015), 12,6\% dos estudantes afirmaram ter desejos de morrer e 5,5\% afirmam em querer fazer algo para tirar as próprias vidas. Esses dados evidenciam jovens que afirmam ter desejo de morrer. A partir desses valores foram constatados que a prevalência de ideação suicida é preocupante em jovens universitários. Uma vez que associado com a ideia do autor Micin et al (2011), estão relacionados à ideação suicida: transtornos de adaptação, humor, transtornos alimentares e ansiedade.

Nesse estudo levantado, constatou-se que em dois artigos há o relato da depressão como fator da ideação suicida. Como observada por Baader et al (2014), a depressão está vinculada a uma maior tendência a ter um comportamento alimentar anormal, o uso nocivo de álcool e ações autodestrutivas. O segundo autor, Shenoy et al (2015), associa a depressão à pressão familiar e problemas financeiros.

A partir da elucidação acima foram destacadas as seguintes condições para a ideação suicida: pressão familiar, abuso de drogas e direção perigosa. No entanto, essa revisão observou que não são causas exclusivas, uma vez que há uma alta prevalência da depressão, problema financeiro, incerteza do mercado de trabalho, falta de apoio social, além da influência do contexto sociocultural.

\section{CONCLUSÃO}

Nos artigos acima foi evidenciado a ideia de suicídio em estudantes universitários, sendo essa submetida a fatores internos, externos e abusivos. Além disso, um outro fator importante está associado a depressão com a ideia de morte. Apesar de todos os riscos discutidos, não foi evidenciado, nos artigos acima, uma estratégia de política pública voltada para a ideia de suicídio, isso se observa, com Pereira et al (2015) no qual são necessários serviços de prevenção e intervenção no nível de ensino superior. Assim sendo, nota-se uma necessidade de estratégia voltada para a prevenção e promoção da saúde mental do jovem estudante, evitando ter um pensamento suicida (THESOLIM et al; 2016). Nota-se que há uma carência de intervenção pública na saúde mental do universitário, deixando-o à mercê de estar desassistido e, com isso, podendo levar a pensamento suicida e possivelmente à morte. 


\section{REFERÊNCIAS}

BAADER, M. Tomas. et al. Prevalencia de Transtornos de La Salud Mental en Estudiantes Universitários y Los Factores de Riesgo Emocionales Asociados. Revista Chilena Neuro-Psiquiatria. v. 52, n. 3, p. 167-176, 17 jul. 2014. [acesso em 24 ago 2016]. Disponível em: < https://scielo.conicyt.cl/pdf/rchnp/v52n3/art04.pdf >

DUTRA, Elza. Suicídio de Universitários: O Vazio Existencial de Jovens na Contemporaneidade.

Estudos e Pesquisa em Psicologia. Rio de Janeiro. v. 12, n.3, p. 924-937, 20 out. 2012. [acesso em 24 ago 2016]. Disponível em:

<http://pepsic.bvsalud.org/pdf/epp/v12n3/v12n3a13.p $\underline{\mathrm{df}}>$

ESKIN, Mehmet. et al. Suicidal Behavior and Psychological Distress in University Students: A 12nation Study. Archives of Suicide Research. n. 0, p. 1-20, 15 mar. 2016.

FARIA, O. Yone; GANDOLFI, Lenora; LEIDES, B. A. Mauro. Prevalence of risk behaviors in young university students. Acta Paul Enferm. Brasilia, v. 27, n. 6, p. 591-5, 2 set. 2014. [acesso em 24 ago 2016]. Disponível em: <

https://www.ncbi.nlm.nih.gov/pubmed/26954847 >

LAMIS, A. Dorian; BALLARD, D. Elizabeth; MAY M. Alexis; DVORAK D. Robert. Depressive

Symptoms and Suicidal Ideation in College Students: The Mediatingand Moderating Rolesof Hopelessness, Alcohol Problems, and Social Support. Journal of Clinical Psychology v. 0, n.0, p. 1-14, 2016 [acesso em 15 set 2016]. Disponível em: < https://www.ncbi.nlm.nih.gov/pubmed/27008096 >

MICIN, Sonia; BAGLADI, Veronica. Salud Mental en Estudiantes Universitarios: Incidencia de Psicopatología y Antecedentes de Conducta Suicida en Población que Acude a un Servicio de Salud Estudiantil. Terapia Psicológica. v. 29, n. 1, p. 53-64, jul. 2011. [acesso em 15 set 2016]. Disponível em: <ttps://scielo.conicyt.cl/pdf/terpsicol/v29n1/art06.pdf>

PEREIRA, Adelino; CARDOSO, Francisco. Suicidal Ideaction in University Students: Prevalence and Association With School and Gender. Paidéia. v. 25, n. 62, p. 299-306, 23 apr. 2015. [acesso em 15 set 2016]. Disponível em: < http://www.scielo.br/pdf/paideia/v25n62/1982-4327paideia-25-62-0299.pdf>
SHENOY, P. Divya; LEE, Christine; TRIEU L. Sang. The Mental Health Status of Single Parent Community College Students in California. Journal of American College Health. v.64, p. 152-156, 7 jul. 2015. [acesso em 20 set 2016]. Disponível em: <

https://www.ncbi.nlm.nih.gov/pubmed/26151464>

SILVA, A. Clóvis. et al. Suicidal Ideation Among Students Enrolled In Healthcare Training Programs: A Cross-Sectional Study. Revista Brasileira

Psiquiatria. v. 31, n. 4, p. 388-44, 26 jun. 2009. [acesso em 20 set 2016. Disponível em: < http://www.scielo.br/pdf/rbp/v31n4/aop0909.pdf >

THESOLIM, L. Bruna; BERNARDINO, Angela; FERREIRA, L. Vitor; BAPTISTA, A. Claudio. Suicídios em Município do Interior de São Paulo: Caracterização e Prevalência de Gêneros. Revista Brasileira Multidisciplinar. v. 19, n. 1, p. 139-146. Jul. 2016. [acesso em 20 set 2016]. Disponível em: < http://www.revistarebram.com/index.php/revistauniara /article/view/372>
Isabella Maria de Oliveira Miranda
Discente do Curso de Medicina do Centro
Universitário das Faculdades Associadas de Ensino.

\section{Eduarda Zeuri}

Discente do Curso de Medicina do Centro Universitário das Faculdades Associadas de Ensino.

Karolina Tank
Discente do Curso de Medicina do Centro
Universitário das Faculdades Associadas de Ensino.

João Gabriel Barbosa
Discente do Curso de Medicina do Centro
Universitário das Faculdades Associadas de Ensino.

\begin{tabular}{l}
\hline Nelson Antônio Filho \\
Mestrado pela Santa Casa de Misericórdia de São \\
Paulo. \\
\hline \\
\hline Laura Ferreira de Rezende \\
Mestre e Doutora pela Faculdade de Ciências \\
Médicas da Universidade de Campinas e pós-doutora \\
pela Universidade do Estado de São Paulo. \\
\hline
\end{tabular}

